\title{
IF topological vector spaces
}

\author{
Moumita Chiney and S. K. Samanta \\ Department of Mathematics, Visva-Bharati \\ Santiniketan 731235, West Bengal, India \\ e-mails: moumi .chiney@gmail.com, \\ syamal_123@yahoo.co.in
}

Received: 8 April 2018

Accepted: 20 April 2018

\begin{abstract}
In the present paper a notion of intuitionistic fuzzy topology on an intuitionistic fuzzy set has been developed. Also the concept of IF topological vector space has been introduced which is a combined structure of intuitionistic fuzzy vector space and intuitionistic fuzzy topology as defined by us. In this study, we generalized the action of a group on a set to intuitionistic fuzzy action. We obtained some basic results.
\end{abstract}

Keywords: Intuitionistic fuzzy sets, Intuitionistic fuzzy vector space, Intuitionistic fuzzy topology on intuitionistic fuzzy set, IF topological vector space.

2010 Mathematics Subject Classification: 03E72, 15A03, 54A05.

\section{Introduction}

Allowing some kind of independence in the falsity value from the truth value, K. T. Atanassov [1-4] proposed the notion of intuitionistic fuzzy set (IFS) which is a generalization of Zadeh's fuzzy set [24]. IFS theory have successfully been applied in knowledge engineering, medical diagnosis, decision making, career determination etc. [13, 14, 23]. Much work have been done in developing various mathematical structures such as groups, rings, topological spaces, metric spaces, topological vector spaces etc. in IFS [6,12, 15, 18-21]. We have introduced a notion of intuitionistic fuzzy vector space, intuitionistic fuzzy basis and intuitionistic fuzzy dimension in $[10,11]$.

In this paper, by synthesizing the definition of fuzzy topologies of Chakraborty and Ahsanullah [8] and of Lowen [17], we extend it to IF setting to introduce a definition of intuitionistic fuzzy topology on intuitionistic fuzzy set. Also we introduce a notion of IF topological vector 
space associated with an intuitionistic fuzzy vector space [10] and intuitionistic fuzzy topology defined on this intuitionistic fuzzy vector space. Some fundamental properties of IF topological vector spaces have been investigated.

\section{Preliminaries}

Definition 2.1 ([1]). Let $X$ be a non-empty set. An intuitionistic fuzzy set (IFS for short) of $X$ is defined as an object having the form $A=\left\{\left\langle x, \mu_{A}(x), \nu_{A}(x)\right\rangle \mid x \in X\right\}$, where $\mu_{A}$ : $X \rightarrow[0,1]$ and $\nu_{A}: X \rightarrow[0,1]$ denote the degree of membership (namely $\mu_{A}(x)$ ) and the degree of non-membership (namely $\nu_{A}(x)$ ) of each element $x \in X$ to the set $A$, respectively, and $0 \leq \mu_{A}(x)+\nu_{A}(x) \leq 1$ for each $x \in X$. For the sake of simplicity we shall use the symbol $A=\left(\mu_{A}, \nu_{A}\right)$ for the intuitionistic fuzzy set $A=\left\{\left\langle x, \mu_{A}(x), \nu_{A}(x)\right\rangle \mid x \in X\right\}$.

Definition 2.2 ([1]). Let $A=\left(\mu_{A}, \nu_{A}\right)$ and $B=\left(\mu_{B}, \nu_{B}\right)$ be intuitionistic fuzzy sets of a set $X$.

Then

(1) $A \subseteq B$ iff $\mu_{A}(x) \leq \mu_{B}(x)$ and $\nu_{A}(x) \geq \nu_{B}(x)$ for all $x \in X$.

(2) $A=B$ iff $A \subseteq B$ and $B \subseteq A$.

(3) $A^{c}=\left\{\left\langle x, \nu_{A}(x), \mu_{A}(x)\right\rangle \mid x \in X\right\}$

(4) $A \cap B=\left\{\left\langle x, \mu_{A}(x) \wedge \mu_{B}(x), \nu_{A}(x) \vee \nu_{B}(x)\right\rangle \mid x \in X\right\}$.

(5) $A \cup B=\left\{\left\langle x, \mu_{A}(x) \vee \mu_{B}(x), \nu_{A}(x) \wedge \nu_{B}(x)\right\rangle \mid x \in X\right\}$.

(6) $\square A=\left\{\left\langle x, \mu_{A}(x), 1-\mu_{A}(x)\right\rangle \mid x \in X\right\}, \diamond A=\left\{\left\langle x, 1-\nu_{A}(x), \nu_{A}(x)\right\rangle \mid x \in X\right\}$.

Definition 2.3 ([4]). Let $A$ be an IFS in a set $X$. Then for $\lambda, \xi \in[0,1]$ with $\lambda+\xi \leq 1$, the set $A^{[\lambda, \xi]}=\left\{x \in X: \mu_{A}(x) \geq \lambda\right.$ and $\left.\nu_{A}(x) \leq \xi\right\}$ is called $(\lambda, \xi)$-level subset of $A$.

Proposition 2.4 ([4]). Let $A$ be an IFS in a set $X$ and $\left(\lambda_{1}, \xi_{1}\right),\left(\lambda_{2}, \xi_{2}\right) \in \operatorname{Im}(A)$. If $\lambda_{1} \leq \lambda_{2}$ and $\xi_{1} \geq \xi_{2}$, then $A^{\left[\lambda_{1}, \xi_{1}\right]} \supseteq A^{\left[\lambda_{2}, \xi_{2}\right]}$.

Definition 2.5 ([5,18]). Let $X$ be a vector space over the field $K$, the field of real and complex numbers, $\alpha \in K, A=\left(\mu_{A}, \nu_{A}\right)$ and $B=\left(\mu_{B}, \nu_{B}\right)$ be two intuitionistic fuzzy sets of $X$. Then

(1) the sum of $A$ and $B$ is defined to be the intuitionistic fuzzy set $A+B=\left(\mu_{A}+\mu_{B}, \nu_{A}+\nu_{B}\right)$ of $X$ given by

$$
\begin{aligned}
& \mu_{A+B}(x)= \begin{cases}\sup _{x=a+b}\left\{\mu_{A}(a) \wedge \mu_{B}(b)\right\} & \text { if } x=a+b \\
0 & \text { otherwise, }\end{cases} \\
& \nu_{A+B}(x)= \begin{cases}\inf \left\{\nu_{A}(a) \vee \nu_{B}(b)\right\} & \text { if } x=a+b \\
x=a+b & \text { otherwise. }\end{cases}
\end{aligned}
$$


(2) $\alpha A$ is defined to be the IFS $\alpha A=\left(\mu_{\alpha A}, \nu_{\alpha A}\right)$ of $X$, where

$$
\begin{aligned}
& \mu_{\alpha A}(x)= \begin{cases}\mu_{A}\left(\alpha^{-1} x\right) & \text { if } \alpha \neq 0 \\
\sup _{y \in X} \mu_{A}(y) & \text { if } \alpha=0, x=\theta \\
0 & \text { if } \alpha=0, x \neq \theta,\end{cases} \\
& \nu_{\alpha A}(x)= \begin{cases}\nu_{A}\left(\alpha^{-1} x\right) & \text { if } \alpha \neq 0 \\
\text { inf } \nu_{A}(y) & \text { if } \alpha=0, x=\theta \\
y \in X & \text { if } \alpha=0, x \neq \theta . \\
1 & \text { in }\end{cases}
\end{aligned}
$$

Definition 2.6 ([10]). An IFS $V=\left(\mu_{V}, \nu_{V}\right)$ of a vector space $X$ over the field $K$ is said to be intuitionistic fuzzy vector space over $X$ if

(i) $V+V \subseteq V$

(ii) $\alpha V \subseteq V$, for every scalar $\alpha$.

We denote the set of all intuitionistic fuzzy vector spaces over a vector space X by IFVS $(X)$.

Lemma 2.7 ([10]). Let $V$ be an intuitionistic fuzzy set in a vector space $X$. Then, the following are equivalent:

(1) $V$ is an intuitionistic fuzzy vector space over $X$.

(2) For all scalars $\alpha, \beta$, we have $\alpha V+\beta V \subseteq V$.

(3) For all scalars $\alpha, \beta$ and for all $x, y \in X$, we have

$$
\left.\mu_{V}(\alpha x+\beta y) \geq \mu_{V}(x) \wedge \mu_{V}(y)\right\} \text { and } \nu_{V}(\alpha x+\beta y) \leq \nu_{V}(x) \vee \nu_{V}(y) .
$$

Remark 2.8 ([10]). Our definition of intuitionistic fuzzy vector space is equivalent to the definition of intuitionistic fuzzy subspace of [21] and [9].

Proposition 2.9 ([10]). Let $V \in \operatorname{IFV} S(X)$. Then $\mu_{V}(\theta) \geq \mu_{V}(x)$ and $\nu_{V}(\theta) \leq \nu_{V}(x), \forall x \in X$.

Definition 2.10 ([10]). For any $(a, b),(c, d) \in[0,1] \times[0,1]$ with $a+b \leq 1, c+d \leq 1$, we say that:

(1) $(a, b) \geq(c, d)$ if $a \geq b$ and $c \leq d$.

(2) $(a, b) \leq(c, d)$ if $a \leq b$ and $c \geq d$.

(3) $(a, b)>(c, d)$ if $a>b$ and $c \leq d$ or if $a \geq b$ and $c<d$.

(4) $(a, b)<(c, d)$ if $a<b$ and $c \geq d$ or if $a \leq b$ and $c>d$.

(5) $(a, b)=(c, d)$ if $a=b$ and $c=d$.

Unless otherwise stated in the rest of the paper the collection of all intuitionistic fuzzy subsets of $X$ is denoted by $\eta^{X}$, where $\eta=\{(k, m) \in[0,1] \times[0,1]: k+m \leq 1\}$. 
Definition 2.11. [12] An intuitionistic fuzzy topology on a non-empty set $X$ is a family $\tau$ of intuitionistic fuzzy sets in $X$ which satisfies the following conditions:

(i) $0_{\sim}, 1_{\sim} \in \tau$;

(ii) If $A, B \in \tau$, then $A \cap B \in \tau$;

(iii) If $A_{i} \in \tau$, for each $i \in I$, then $\bigcup_{i \in I} \in \tau$.

In this case $(X, \tau)$ is called an intuitionistic fuzzy topological space. The members of $\tau$ are called the intuitionistic fuzzy open sets and the complement $A^{C}$ of an intuitionistic fuzzy open set in an intuitionistic fuzzy topological space $(X, \tau)$ is called an intuitionistic fuzzy closed set.

Definition $2.12([12])$. Let $(X, \tau)$ and $(Y, \delta)$ be two intuitionistic fuzzy topological spaces and let $f: X \rightarrow Y$ be a function. Then $f$ is said to be an intuitionistic fuzzy continuous if the pre-image of each intuitionistic fuzzy set of $\delta$ is an intuitionistic fuzzy set in $\tau$.

Definition 2.13 ([22]). Let $(r, s),(p, q) \in \eta$. Define

(i) $(r, s) \sqcup(p, q)=(r \vee p, s \wedge q)$,

(ii) $(r, s) \sqcap(p, q)=(r \wedge p, s \vee q)$,

where $\vee$ and $\wedge$ are usual maximum and minimum inthe ordered set of real numbers.

Definition 2.14 ([22]). Let $X$ and $Y$ be two non-empty sets and let $A \in \eta^{X}$ and $B \in \eta^{Y}$. An intuitionistic fuzzy subset $F$ of $X \times Y$ is said to be an intuitionistic fuzzy proper function from the intuitionistic fuzzy set $A$ to the intuitionistic fuzzy set $B$ if

(i) $F(x, y) \leq A(x) \sqcap B(y)$, for each $(x, y) \in X \times Y$.

(ii) for each $x \in X$, there exists a unique $y_{0} \in Y$ such that $F\left(x, y_{0}\right)=A(x)$ and $F(x, y)=$ $(0,1)$, if $y \neq y_{0}$.

Henceforth $F: A \rightarrow B$ implies $F$ is an intuitionistic fuzzy proper function from $A \in \eta^{X}$ into $B \in \eta^{Y}$.

Definition 2.15 ([22]). Let $F: A \rightarrow B$. If $U \subset A$ and $V \subset B$, then $F^{-1}(V) \subset A$ and $F(U) \subset B$ are defined by

(i) $F^{-1}(V)(x)=\underset{s \in Y}{\sqcup}\{F(x, s) \sqcap V(s)\}, \forall x \in X$.

(ii) $F(U)(y)=\bigsqcup_{t \in X}\{F(t, y) \sqcap U(t)\}, \forall y \in Y$.

Lemma 2.16 ([22]). Let $F: A \rightarrow B$ be an intuitionistic fuzzy proper function. If $V \subset B$, then $F^{-1}(V)(x)=A(x) \sqcap V(y)$, where $y \in Y$ is unique such that $F(x, y)=A(x)$.

Lemma 2.17 ([22]). Let $F: A \rightarrow B$ be an intuitionistic fuzzy proper function and $U \subset A$, $V \subset B$. Then $F\left(F^{-1}(V)\right) \subset V$ and $U \subset F^{-1}(F(U))$. 
Definition 2.18 ([22]). $A \times B: X \times Y \rightarrow \eta$ is defined by $A \times B(x, y)=A(x) \sqcap B(y)$, $\forall(x, y) \in X \times Y$.

Definition 2.19 ([22]). The intuitionistic fuzzy proper function $p_{A}: A \times B \rightarrow A$ defined by $p_{A}((x, y), z)=(A \times B)(x, y)$ or $(0,1)$ accordingly as $z=x$ or $z \neq x$ $\forall x, z \in X$ and $\forall y \in Y$ is said to be the intuitionistic fuzzy projection map of $A \times B$ into $A$. Similarly, the intuitionistic fuzzy projection map $p_{B}: A \times B \rightarrow B$ is defined.

Lemma 2.20 ([22]). Let $U \subset A, V \subset B$. Then $p_{A}^{-1}(U)=U \times B, p_{B}^{-1}(V)=A \times V$.

\section{Intuitionistic fuzzy topology on intuitionistic fuzzy set}

Definition 3.1. An intuitionistic fuzzy set $A$ of $X$ is said to be constant which will be denoted by $(k, m)_{\sim}$ and defined by $\mu_{A}(x)=k$ and $\nu_{A}(x)=m,(k, m) \in \eta, \forall x \in X$.

Definition 3.2. Let $A$ be a intuinistic fuzzy subset of $X$. A collection $\tau$ of intuitionistic fuzzy subsets of $A$ satisfying

(i) $(k, m)_{\sim} \cap A \in \tau, \forall(k, m) \in \eta$;

(ii) If $A, B \in \tau$, then $A \cap B \in \tau$;

(iii) If $A_{i} \in \tau$, for each $i \in I$, then $\cup_{i \in I} \in \tau$.

is called an intuitionistic fuzzy topology or IF topology on the intuitionistic fuzzy set A. The pair $(A, \tau)$ is called an intuitionistic fuzzy topological space. Members of $\tau$ will be called intuitionistic fuzzy open sets.

Unless otherwise mentioned by an intuitionistic fuzzy topological space we shall mean it in the sense of Definition 3.2 and $(A, \tau)$ will denote an intuitionistic fuzzy topological space.

Proposition 3.3. If $\mathcal{B}$ be a given collection of intuitionistic fuzzy subsets of an intuitionistic fuzzy set $A$ and the family $\{(k, m) \sim A \in \tau,(k, m) \in \eta\}$, then the family of all possible unions and finite intersections of the members of $\mathcal{B}$ is an intuitionistic fuzzy topology on $A$ and it will be denoted by $\tau(\mathcal{B})$.

Definition 3.4. $\mathcal{B} \subset \tau$ is called an open base of $\tau$ if every member of $\tau$ can be expressed as a union of some members of $\mathcal{B}$.

Definition 3.5. An intuitionistic fuzzy proper function $F: A \rightarrow B$ is said to be

(i) injective if $F\left(x_{1}, y\right)=A\left(x_{1}\right)(\neq(0,1)), F\left(x_{2}, y\right)=A\left(x_{2}\right)(\neq(0,1)) \Rightarrow x_{1}=x_{2}, \forall x_{1}, x_{2} \in$ $X, y \in Y$

(ii) surjective if $\forall y \in Y$ with $B(y) \neq(0,1), \exists x \in X$ such that $F(x, y)=A(x)=B(y)$;

(iii) bijective if $F$ is both injective and surjective. 
Proposition 3.6. If $F: A \rightarrow B$ is injective, then for all $V \subseteq A, F^{-1}(F(V))=V$.

Proof. Let $x \in X$ and $y$ be unique such that $F(x, y)=A(x)$.

Then $\left[F^{-1}(F(V))\right](x)=\underset{s \in Y}{\sqcup}\{F(x, s) \sqcap F(V)(s)\}$

$$
\begin{aligned}
& =F(x, y) \sqcap F(V)(y) \\
& =A(x) \sqcap \underset{t \in X}{\sqcup}\{F(t, y) \sqcap V(y)\} \\
& =A(x) \sqcap A(x) \sqcap V(x) \text { [Since } F \text { is injective] } \\
& =V(x) .
\end{aligned}
$$

Proposition 3.7. If $F: A \rightarrow B$ is surjective, then $F(V)=W$ and for all $W \subseteq B, F\left(F^{-1}(W)\right)=$ $W$.

Proof. For any $y \in Y$ with $B(y)=(0,1)$ implies $F(A)(y)=(0,1)$. Hence for those $y \in Y$, $F(A)(y)=B(y)$.

For any $y \in Y$ with $B(y) \neq(0,1)$,

$F(A)(y)=\sqcup_{t \in X}\{F(t, y) \sqcap A(t)\}$

$=\sqcup\{A(x): x \in X$ with $F(x, y)=A(x)=B(y)\}$ [Since $F$ is surjective]

$=B(y)$.

Hence $F(A)=B$.

For any $W \subseteq B, F\left(F^{-1}(W)\right)(y)=\sqcup_{x \in X}\left\{F(x, y) \sqcap F^{-1}(W)(x)\right\}$

$=\sqcup\left\{A(x) \sqcap F^{-1}(W)(x): x \in X, F(x, y)=A(x)=B(y)\right\}$, [since $F$ is surjective]

$=\sqcup\left\{F^{-1}(W)(x): x \in X, F(x, y)=A(x)=B(y)\right\}$

$\sqcup\{A(x) \sqcap W(y): x \in X, F(x, y)=A(x)=B(y)\}$, [By Proposition 2.16]

$\sqcup\{B(y) \sqcap W(y): x \in X, F(x, y)=A(x)=B(y)\}$

$=W(y)$.

Hence $F\left(F^{-1}(W)\right)=W$.

Proposition 3.8. Let $F: A \rightarrow B$ be an intuitionistic fuzzy proper function. If $V, W \subseteq A$, then

(i) $V \subseteq W \Longrightarrow F(V) \subseteq F(W)$.

(ii) $F(V \cup W)=F(V) \cup F(W)$.

(iii) $F(V \cap W) \subseteq F(V) \cap F(W)$.

(iv) $F(V \cap W)=F(V) \cap F(W)$, if $F$ is injective.

Proof. (i) is obvious.

(ii) For any $y \in Y$,

$F(V \cup W)(y)=\sqcup_{x \in X}\{F(x, y) \sqcap(V \cup W)(x)\}$

$=\sqcup\{A(x) \sqcap(V \cup W)(x): x \in X$ such that $F(x, y)=A(x)\}$

$=\sqcup\{[A(x) \sqcap V(x)] \sqcup[A(x) \sqcap W(x)]: x \in X$ such that $F(x, y)=A(x)\}$

$=[\sqcup\{[A(x) \sqcap V(x)]: x \in X$ such that $F(x, y)=A(x)\}] \sqcup[\sqcup\{[A(x) \sqcap W(x)]: x \in$ $X$ such that $F(x, y)=A(x)\}]$ 
$=[F(V) \cup F(W)](y)$.

Therefore, $F(V \cup W)=F(V) \cup F(W)$.

(iii) For any $y \in Y$,

$[F(V) \cap F(W)](y)=[\sqcup\{[A(x) \sqcap V(x)]: x \in X$ such that $F(x, y)=A(x)\}] \sqcap[\sqcup\{[A(x) \sqcap$ $W(x)]: x \in X$ such that $F(x, y)=A(x)\}]$

$[\sqcup\{V(x): x \in X$ such that $F(x, y)=A(x)\}] \sqcap[\sqcup\{W(x): x \in X$ such that $F(x, y)=A(x)\}]$

$\geq \sqcup\{V(x) \sqcap W(x): x \in X$ such that $F(x, y)=A(x)\}$

$=\sqcup\{A(x) \sqcap[V(x) \sqcap W(x)]: x \in X$ such that $F(x, y)=A(x)\}$

$=F(V \cap W)(y)$.

Therefore, $F(V \cap W) \subseteq F(V) \cap F(W)$.

(iv) If $F$ is injective, for any $y \in Y$,

$[F(V) \cap F(W)](y)=V(x) \sqcap W(x)$, for $x \in X$ unique such that $F(x, y)=A(x)$

$=F(V \cap W)(y)$.

Hence $F(V \cap W)=F(V) \cap F(W)$.

Proposition 3.9. Let $F: A \rightarrow B$ be an intuitionistic fuzzy proper function. If $V, W \subseteq B$, then

(i) $V \subseteq W \Longrightarrow F^{-1}(V) \subseteq F^{-1}(W)$.

(ii) $F^{-1}(V \cup W)=F^{-1}(V) \cup F^{-1}(W)$.

(iii) $F^{-1}(V \cap W)=F^{-1}(V) \cap F^{-1}(W)$.

Proof. (i) is obvious.

(ii) For any $x \in X$,

$F^{-1}(V \cup W)(x)=\sqcup_{y \in Y}\{F(x, y) \sqcap(V \cup W)(y)\}$

$=A(x) \sqcap(V \cup W)(y)$, for $y \in Y$ unique such that $F(x, y)=A(x)$;

$=[A(x) \sqcap V(y)] \sqcup[A(x) \sqcap W(y)]$, for $y \in Y$ unique such that $F(x, y)=A(x)$;

$=\left[F^{-1}(V) \cup F^{-1}(W)\right](x)$.

Therefore, $F^{-1}(V \cup W)=F^{-1}(V) \cup F^{-1}(W)$.

(iii) For any $x \in X$,

$F^{-1}(V \cap W)(x)=\sqcup_{y \in Y}\{F(x, y) \sqcap(V \cap W)(y)\}$

$=A(x) \sqcap(V \cap W)(y)$, for $y \in Y$ unique such that $F(x, y)=A(x)$;

$=[A(x) \sqcap V(y)] \sqcap[A(x) \sqcap W(y)]$, for $y \in Y$ unique such that $F(x, y)=A(x)$;

$=\left[F^{-1}(V) \cap F^{-1}(W)\right](x)$.

Therefore, $F^{-1}(V \cap W)=F^{-1}(V) \cap F^{-1}(W)$.

Definition 3.10. The intuitionistic fuzzy proper function $I_{A}: A \rightarrow A$ defined by $I_{A}(x, y)=A(x)$ or $(0,1)$ according as $y=x$ or $y \neq x$ is said to be the identity proper function on $A$. 
Definition 3.11. If $F: A \rightarrow B$ and $G: B \rightarrow C\left(C \in \eta^{Z}\right)$ are intuitionistic fuzzy proper functions, then the intuitionistic fuzzy proper function $G \circ F: A \rightarrow C$ is defined by $G \circ F(x, z)=$ $\begin{cases}A(x), & \text { if } \exists y \text { such that } F(x, y)=A(x), G(y, z)=B(y) \\ (0,1), & \text { otherwise. }\end{cases}$

Definition 3.12. An intuitionistic fuzzy proper function $G: B \rightarrow A$ is called an inverse of a bijective proper function $F: A \rightarrow B$ if $G \circ F=I_{A}$ and $F \circ G=I_{B}$.

Therefore for a bijective intuitionistic fuzzy proper function $F: A \rightarrow B$ defined as in 2.14, its inverse $G: B \rightarrow A$ is defined by

(i) $G(y, x) \leq B(y) \sqcap A(x)$;

(ii) for each $y \in Y$, there is unique $x \in X$ such that $G(y, x)=B(y)$ for $F(x, y)=A(x)$ and $G(y, x)=(0,1)$ otherwise.

Definition 3.13. An intuitionistic fuzzy proper function $F:(A, \tau) \rightarrow\left(B, \tau_{1}\right)$ is said to be

(i) intuitionistic fuzzy continuous if $F^{-1}(V) \in \tau, \forall V \in \tau_{1}$,

(ii) intuitionistic fuzzy open if $F(U) \in \tau_{1}, \forall U \in \tau$,

(iii) intuitionistic fuzzy homeomorphism if $F$ is bijective, intuitionistic fuzzy continuous and inverse of $F$ is also intuitionistic fuzzy continuous.

Proposition 3.14. Let $A \in \eta^{X}, B \in \eta^{Y}$ and $C \in \eta^{Z}$. If $F:(A, \tau) \rightarrow\left(B, \tau_{1}\right)$ and $G:\left(B, \tau_{1}\right) \rightarrow$ $\left(C, \tau_{2}\right)$ are intuitionistic fuzzy continuous proper functions, then the intuitionistic fuzzy proper function $G \circ F:(A, \tau) \rightarrow\left(C, \tau_{2}\right)$ as defined in 3.11 is also intuitionistic fuzzy continuous.

Proof. Let $C_{1} \subseteq C$. Now, $\left[(G \circ F)^{-1}\left(C_{1}\right)\right](x)=\underset{s \in Z}{\sqcup}\left[(G \circ F)(x, s) \sqcap C_{1}(s)\right]$ $= \begin{cases}A(x) \sqcap C_{1}\left(s_{1}\right), & \text { if } \exists y \in Y, s_{1} \in Z \text { such that } F(x, y)=A(x) \text { and } G\left(y, s_{1}\right)=B(y) \\ (0,1), & \text { otherwise. }\end{cases}$ Again $\left[G^{-1}\left(C_{1}\right)\right](y)=\underset{s \in Z}{\sqcup}\left[G(y, s) \sqcap C_{1}(s)\right]$

$=B(y) \sqcap C_{1}\left(s_{y}\right)$, where $s_{y} \in Y$ unique such that $G\left(y, s_{y}\right)=B(y)$.

Thus $\left[F^{-1}\left(G^{-1}\left(C_{1}\right)\right)\right](x)=\sqcup_{t \in Y}\left[F(x, t) \sqcap G^{-1}\left(C_{1}\right)(t)\right]$

$=\sqcup_{t \in Y}\left[F(x, t) \sqcap B(t) \sqcap G^{-1}\left(C_{1}\right)\left(s_{t}\right)\right]$, where $s_{t} \in Y$ unique such that $G\left(t, s_{t}\right)=B(t)$.

$= \begin{cases}A(x) \sqcap C_{1}\left(s_{t^{\prime}}\right), & \text { if } \exists t^{\prime} \in Y, s_{t^{\prime}} \in Z \text { such that } F\left(x, t^{\prime}\right)=A(x) \text { and } G\left(t^{\prime}, s_{t^{\prime}}\right)=B\left(t^{\prime}\right) \\ (0,1), & \text { otherwise. }\end{cases}$

Hence $(G \circ F)^{-1}\left(C_{1}\right)=F^{-1}\left(G^{-1}\left(C_{1}\right)\right)$. Since $G$ and $F$ are intuitionistic fuzzy continuous, for any $C_{1} \in \tau_{2}, G^{-1}\left(C_{1}\right) \in \tau_{1}$ and $F^{-1}\left(G^{-1}\left(C_{1}\right)\right) \in \tau$. Hence $G \circ F$ is intuitionistic fuzzy continuous.

Definition 3.15. An element $a \in X$ is called a normal element of $A$ with respect to $B$ if $A(a) \geq$ $B(y), \forall y \in Y$. 
Lemma 3.16. If $(A, \tau)$ and $\left(B, \tau_{1}\right)$ are intuitionistic fuzzy topological spaces and ' $a$ ' be a normal element of $B$ with respect to $A$, then the intuitionistic fuzzy proper function $F:(A, \tau) \rightarrow\left(B, \tau_{1}\right)$ defined by $F(x, y)= \begin{cases}A(x) & \text { if } y=a \\ (0,1) & \text { if } y \neq a\end{cases}$ is intuitionistic fuzzy continuous.

Proof. Let $V \in \tau_{1}$. Then $\forall x \in X, F^{-1}(V)(x)=\underset{y \in Y}{\sqcup}\{F(x, y) \sqcap V(y)\}=A(x) \sqcap V(a)$, [By definition].

Therefore $F^{-1}(V)=A \cap(k, m)_{\sim} \in \tau$, where $(k, m)=V(a)$. Hence proved.

Lemma 3.17. $U \subset A \in \eta^{X}, V \subset B \in \eta^{Y}$. Then $p_{A}(U \times V)=U \cap(k, m)_{\sim}$, where $(k, m)=$ $\sup \{V(y): y \in Y\}$ and $p_{B}(U \times V)=V \cap\left(k_{1}, m_{1}\right)$, where $\left(k_{1}, m_{1}\right)=\sup \{U(x): x \in X\}$.

Proof. For any $z \in X$,

$p_{A}(U \times V)(z)$

$=\underset{(x, y) \in X \times Y}{\sqcup}\left\{p_{A}((x, y), z) \sqcap(U \times V)(x, y)\right\}$

$=\underset{y \in Y}{\sqcup}\{(A \times B)(z, y) \sqcap(U \times V)(z, y)\}$

$=\underset{y \in Y}{\sqcup}\{(U \times V)(z, y)\}$

$=U(z) \sqcap \underset{y \in Y}{\sqcup}\{V(y)\}$.

Hence $p_{A}(U \times V)=U \cap(k, m)_{\sim}$, where $(k, m)=\sup \{V(y): y \in Y\}$.

Similarly it can be proved that $p_{B}(U \times V)=V \cap\left(k_{1}, m_{1}\right)_{\sim}$, where $\left(k_{1}, m_{1}\right)=\sup \{U(x): x \in$ $X\}$.

Remark 3.18. $p_{A}(A \times B)$ ( or $\left.p_{B}(A \times B)\right)$ may not be equal to $A($ or $B)$. However, if there exists a normal element of $B$ (or $A)$ with respect to $A($ or $B)$, then $p_{A}(A \times B)=A\left(\right.$ or $\left.p_{B}(A \times B)=B\right)$.

Proposition 3.19. The collection $\mathcal{B}=\left\{U \times V: U \in \tau, V \in \tau_{1}\right\}$ forms an open base of an intuitionistic fuzzy topology on $A \times B$.

Proof. For $(k, l) \in \eta,(k, l)_{\sim} \cap(A \times B)=\left((k, l)_{\sim} \cap A\right) \times\left((k, l)_{\sim} \cap B\right) \in \tau \times \tau_{1}$. Hence $(k, l)_{\sim} \cap(A \times B) \in \mathcal{B}$, for all $(k, l) \in \eta$.

Let $U_{1} \times V_{1}, U_{2} \times V_{2} \in \mathcal{B}$. Then $U_{1}, U_{2} \in \tau, V_{1}, V_{2} \in \tau_{1}$.

Now $\left(U_{1} \times V_{1}\right) \cap\left(U_{2} \times V_{2}\right)=\left(U_{1} \cap U_{2}\right) \times\left(V_{1} \cap V_{2}\right) \in \mathcal{B}$.

Therefore $\mathcal{B}$ forms an open base for an intutionistic fuzzy topology on $A \times B$.

Definition 3.20. The intutionistic fuzzy topology in $A \times B$ induced by $\mathcal{B}=\{U \times V: U \in \tau, V \in$ $\left.\tau_{1}\right\}$ is called the product intuitionistic fuzzy topology of $\tau$ and $\tau_{1}$ and is denoted by $\tau \times \tau_{1}$. The intuitionistic fuzzy topological space $\left(A \times B, \tau \times \tau_{1}\right)$ is called the product of the intuitionistic fuzzy topological spaces $(A, \tau)$ and $\left(B, \tau_{1}\right)$.

Theorem 3.21. $p_{A}:\left(A \times B, \tau \times \tau_{1}\right) \rightarrow(A, \tau)$ and $p_{B}:\left(A \times B, \tau \times \tau_{1}\right) \rightarrow\left(B, \tau_{1}\right)$ are intuitionistic fuzzy continuous and intuitionistic fuzzy open. $\tau \times \tau_{1}$ is the smallest intuitionistic fuzzy topology in $A \times B$ with respect to which $p_{A}$ and $p_{B}$ are intuitionistic fuzzy continuous. 
Proof. The $p_{A}$ and $p_{B}$ are intuitionistic fuzzy continuous and open follows from Lemma 2.20 and 3.17. That $\tau \times \tau_{1}$ is the smallest intuitionistic fuzzy topology in $A \times B$ with respect to which $p_{A}$ and $p_{B}$ are intuitionistic fuzzy continuous follows from the fact that if $U \in \tau, V \in \tau_{1}$, then $U \times V=p_{A}^{-1}(U) \cap p_{B}^{-1}(V)$.

Lemma 3.22. If ' $a$ ' be a normal element of $B$ with respect to $A$, then the intuitionistic fuzzy proper function $F_{a}:(A, \tau) \rightarrow\left(A \times B, \tau \times \tau_{1}\right)$ defined by

$F_{a}\left(x,\left(x_{1}, y_{1}\right)\right)= \begin{cases}A(x) & \text { if }\left(x_{1}, y_{1}\right)=(x, a) \\ (0,1) & \text { if }\left(x_{1}, y_{1}\right) \neq(x, a)\end{cases}$

is intuitionistic fuzzy continuous.

Proof. Let $V \times V^{\prime} \in \tau \times \tau_{1}$ and $x \in X$. Then

$F_{a}^{-1}\left(V \times V^{\prime}\right)(x)=\underset{\left(x_{1}, y_{1}\right) \in X \times Y}{\sqcup}\left\{F_{a}\left(x,\left(x_{1}, y_{1}\right)\right) \sqcap\left(V \times V^{\prime}\right)\left(x_{1}, y_{1}\right)\right\}$

$=A(x) \sqcap\left(V \times V^{\prime}\right)(x, a)$

$=A(x) \sqcap(k, m) \sqcap V(x)$, [where $V^{\prime}(a)=(k, m)$ ].

Therefore $F_{a}^{-1}\left(V \times V^{\prime}\right)=A \cap(k, m)_{\sim} \cap V \in \tau$. Hence $F_{a}$ is intuitionistic fuzzy continuous.

Similarly we have,

Lemma 3.23. If ' $a$ ' be a normal element of $B$ with respect to $A$, then the intuitionistic fuzzy proper function $F_{a}:(A, \tau) \rightarrow\left(B \times A, \tau_{1} \times \tau\right)$ defined by

$F_{a}\left(x,\left(y_{1}, x_{1}\right)\right)= \begin{cases}A(x) & \text { if }\left(y_{1}, x_{1}\right)=(a, x) \\ (0,1) & \text { if }\left(y_{1}, x_{1}\right) \neq(a, x)\end{cases}$

is intuitionistic fuzzy continuous.

Theorem 3.24. Let $\left(A_{i}, \tau_{i}\right)$ and $\left(B_{i}, \sigma_{i}\right), i=1,2$ be intuitionistic fuzzy topological spaces and $F_{i}:\left(A_{i}, \tau_{i}\right) \rightarrow\left(B_{i}, \sigma_{i}\right), i=1,2$ be intuitionistic fuzzy continuous proper functions, where $A_{i} \in$ $\eta^{X_{i}}, B_{i} \in \eta^{Y_{i}}$. Then for each $i=1,2, x_{i} \in X_{i}, \exists$ unique $y_{i_{0}} \in Y_{i}$ such that $F_{i}\left(x_{i}, y_{i_{0}}\right)=A_{i}\left(x_{i}\right)$ and $F_{i}\left(x_{i}, y_{i}\right)=(0,1)$ if $y_{i} \neq y_{i_{0}}$. Now if we define the proper function $F=F_{1} \times F_{2}: A_{1} \times A_{2} \rightarrow$ $B_{1} \times B_{2}$ by

$F\left(\left(x_{1}, x_{2}\right),\left(y_{1}, y_{2}\right)\right)= \begin{cases}\left(A_{1} \times A_{2}\right)\left(x_{1}, x_{2}\right) & \text { if }\left(y_{1}, y_{2}\right)=\left(y_{1_{0}}, y_{2_{0}}\right) \\ (0,1) & \text { if }\left(y_{1}, y_{2}\right) \neq\left(y_{1_{0}}, y_{2_{0}}\right)\end{cases}$

is also intuitionistic fuzzy continuous.

Proof. Let $U \times V \in \sigma_{1} \times \sigma_{2}$. Then $\forall\left(x_{1}, x_{2}\right) \in X_{1} \times X_{2}$,

$\left(F_{1} \times F_{2}\right)^{-1}(U \times V)\left(x_{1}, x_{2}\right)$

$=\underset{\left(y_{1}, y_{2}\right) \in Y_{1} \times Y_{2}}{\sqcup}\left\{F\left(\left(x_{1}, x_{2}\right),\left(y_{1}, y_{2}\right)\right) \sqcap(U \times V)\left(\left(y_{1}, y_{2}\right)\right)\right\}$

$=\left[\left(A_{1} \times A_{2}\right)\left(x_{1}, x_{2}\right)\right] \sqcap\left[(U \times V)\left(y_{1_{0}}, y_{2_{0}}\right)\right]$

$=\left(A_{1}\left(x_{1}\right) \sqcap U\left(y_{1_{0}}\right)\right) \times\left(A_{2}\left(x_{2}\right) \sqcap V\left(y_{2_{0}}\right)\right)$.

Now, $\left(F_{1}^{-1}(U) \times F_{2}^{-1}(V)\right)\left(x_{1}, x_{2}\right)$

$=\underset{y_{1} \in Y_{1}}{\sqcup}\left\{F_{1}\left(x_{1}, y_{1}\right) \sqcap U\left(y_{1}\right)\right\} \sqcap \underset{y_{2} \in Y_{2}}{\sqcup}\left\{F_{2}\left(x_{2}, y_{2}\right) \sqcap V\left(y_{2}\right)\right\}$

$=\left(A_{1}\left(x_{1}\right) \sqcap U\left(y_{1_{0}}\right)\right) \times\left(A_{2}\left(x_{2}\right) \sqcap V\left(y_{2_{0}}\right)\right)$.

Therefore $\left(F_{1} \times F_{2}\right)^{-1}(U \times V)=F_{1}^{-1}(U) \times F_{2}^{-1}(V)$. Since $F_{1}$ and $F_{2}$ are intuitionistic fuzzy 
continuous, $F_{1}^{-1}(U) \times F_{2}^{-1}(V)$ is intuitionistic fuzzy open set in $\tau_{1} \times \tau_{2}$. Hence $F=F_{1} \times F_{2}$ is intuitionistic fuzzy continuous.

Theorem 3.25. Let $\left(A_{i}, \tau_{i}\right)$ and $\left(B_{i}, \sigma_{i}\right), i=1,2$ be intuitionistic fuzzy topological spaces and $F_{i}:\left(A_{i}, \tau_{i}\right) \rightarrow\left(B_{i}, \sigma_{i}\right), i=1,2$ be injective intuitionistic fuzzy open proper functions, where $A_{i} \in \eta^{X_{i}}, B_{i} \in \eta^{Y_{i}}$. Then for each $i=1,2, x_{i} \in X_{i}, \exists$ unique $y_{i_{0}} \in Y_{i}$ such that $F_{i}\left(x_{i}, y_{i_{0}}\right)=$ $A_{i}\left(x_{i}\right)$ and $F_{i}\left(x_{i}, y_{i}\right)=(0,1)$ if $y_{i} \neq y_{i_{0}}$. Now if we define the proper function $F=F_{1} \times F_{2}$ : $A_{1} \times A_{2} \rightarrow B_{1} \times B_{2}$ by

$F\left(\left(x_{1}, x_{2}\right),\left(y_{1}, y_{2}\right)\right)= \begin{cases}\left(A_{1} \times A_{2}\right)\left(x_{1}, x_{2}\right) & \text { if }\left(y_{1}, y_{2}\right)=\left(y_{1_{0}}, y_{2_{0}}\right) \\ (0,1) & \text { if }\left(y_{1}, y_{2}\right) \neq\left(y_{1_{0}}, y_{2_{0}}\right)\end{cases}$

is also intuitionistic fuzzy open.

Proof. Let $U \times V \in \tau_{1} \times \tau_{2}$. Then $\forall\left(y_{1}, y_{2}\right) \in Y_{1} \times Y_{2}$,

$\left(F_{1} \times F_{2}\right)(U \times V)\left(y_{1}, y_{2}\right)$

$=\underset{\left(x_{1}, x_{2}\right) \in\left(X_{1} \times X_{2}\right)}{\sqcup}\left\{F\left(\left(x_{1}, x_{2}\right),\left(y_{1}, y_{2}\right)\right) \sqcap(U \times V)\left(x_{1}, x_{2}\right)\right\}$

$=\left(A_{1} \times A_{2}\right)\left(x_{1}, x_{2}\right) \sqcap(U \times V)\left(x_{1}, x_{2}\right)$, for $\left(x_{1}, x_{2}\right) \in X_{1} \times X_{2}$ such that

$F\left(\left(x_{1}, x_{2}\right),\left(y_{1}, y_{2}\right)\right)=\left(A_{1} \times A_{2}\right)\left(x_{1}, x_{2}\right)$

$=(U \times V)\left(x_{1}, x_{2}\right)$, for $\left(x_{1}, x_{2}\right) \in X_{1} \times X_{2}$ such that $F\left(\left(x_{1}, x_{2}\right),\left(y_{1}, y_{2}\right)\right)=\left(A_{1} \times A_{2}\right)\left(x_{1}, x_{2}\right)$.

Again, $\left[F_{1}(U) \times F_{2}(V)\right]\left(y_{1}, y_{2}\right)$

$=\left[\underset{x_{1} \in X_{1}}{\sqcup}\left\{F_{1}\left(x_{1}, y_{1}\right) \sqcap U\left(x_{1}\right)\right\}\right] \sqcap\left[{ }_{x_{2} \in X_{2}}\left\{F_{2}\left(x_{2}, y_{2}\right) \sqcap V\left(x_{2}\right)\right\}\right]$

$=\left[A_{1}\left(x_{1}\right) \sqcap U\left(x_{1}\right)\right] \sqcap\left[A_{2}\left(x_{2}\right) \sqcap V\left(x_{2}\right)\right]$, for $x_{1} \in X_{1}$ such that $F_{1}\left(x_{1}, y_{1}\right)=A_{1}\left(x_{1}\right)$ and $x_{2} \in X_{2}$ such that $F_{2}\left(x_{2}, y_{2}\right)=A_{2}\left(x_{2}\right)$

$=U\left(x_{1}\right) \sqcap V\left(x_{2}\right)$, for $x_{1} \in X_{1}$ such that $F_{1}\left(x_{1}, y_{1}\right)=A_{1}\left(x_{1}\right)$ and $x_{2} \in X_{2}$ such that $F_{2}\left(x_{2}, y_{2}\right)=$ $A_{2}\left(x_{2}\right)$

$=(U \times V)\left(x_{1}, x_{2}\right)$, for $\left(x_{1}, x_{2}\right) \in X_{1} \times X_{2}$ such that $F\left(\left(x_{1}, x_{2}\right),\left(y_{1}, y_{2}\right)\right)=\left(A_{1} \times A_{2}\right)\left(x_{1}, x_{2}\right)$. Therefore $\left(F_{1} \times F_{2}\right)(U \times V)=F_{1}(U) \times F_{2}(V) \in \sigma_{1} \times \sigma_{2}$.

Hence $F=F_{1} \times F_{2}$ is intuitionistic fuzzy open.

\section{IF topological vector space}

Definition 4.1 ([17]). Given a topological space $(X, \tau)$, the collection $\omega(\tau)$, of all fuzzy sets in $X$ which are lower semi-continuous, as functions from $X$ to the unit interval equipped with the usual topology, is a fuzzy topology on X. This fuzzy topology $\omega(\tau)$ is said to be the fuzzy topology generated by the topology $\tau$.

Definition 4.2 ([16]). Let $\mathbb{K}$ be the field of real or complex numbers. Then the fuzzy usual topology on $\mathbb{K}$ is the fuzzy topology generated by the usual topology on $\mathbb{K}$.

Throughout the section we consider $V$ as an intuitionistic fuzzy vector space associated with a vector space $X$ and the ground field $\mathbb{K}$. We consider $\mathbb{K}$ to be equipped with the fuzzy usual topology $\nu$ as defined in Definition 4.2. 
Definition 4.3. Let $X$ be a vector space over the field $\mathbb{K}$ with $\theta$ as the null vector. Let $V$ be an intuitionistic fuzzy vector space over $X, a \in X$ and $k_{0} \in \mathbb{K}$ be fixed. Let us define the intuitionistic fuzzy proper functions

$$
\begin{aligned}
& F^{\oplus}: V \times V \rightarrow V \text { by } F^{\oplus}((x, y), z)= \begin{cases}(V \times V)(x, y) & \text { if } x+y=z \\
(0,1) & \text { if } x+y \neq z,\end{cases} \\
& F^{a}: V \rightarrow V \text { by } F^{a}(x, y)=\left\{\begin{array}{l}
V(x) \text { if } y=a+x \\
(0,1) \text { if } y \neq a+x
\end{array} ;\right. \\
& F^{\odot}:(\mathbb{K} \times V) \rightarrow V \text { by } F^{\odot}((k, x), y)= \begin{cases}\mathbb{K} \times V(k, x) & \text { if } k x=y, k \neq 0 \\
\sup V(x) & \text { if } k x=y, k=0 ; \\
x \in X & \text { if } k x \neq y \\
(0,1) & \end{cases}
\end{aligned}
$$$$
F^{k_{0}}: V \rightarrow V \text { by } F^{k_{0}}(x, y)=\left\{\begin{array}{ll}
V(x) & \text { if } y=k_{0} x, k_{0} \neq 0 \\
\sup _{x \in X} V(x) & \text { if } k_{0} x=y, k_{0}=0 \\
(0,1) & \text { if } y \neq k_{0} x
\end{array}\right. \text {; }
$$$$
F_{V}^{L_{(k, m)}}: V \times V \rightarrow V \text { by }
$$$$
F_{V}^{L_{(k, m)}}((x, y), z)= \begin{cases}(V \times V)(x, y) & \text { if } k x+m y=z, k \neq 0, m \neq 0 \\ V(x) & \text { if } z=k x, k \neq 0, m=0 \\ V(y) & \text { if } z=m y, k=0, m \neq 0 \\ \sup _{s \in X} V(s) & \text { if } k x+m y=z, k=0, m=0 \\ (0,1) & \text { if } k x+m y \neq z,\end{cases}
$$

for all $x, y, z \in X, k, m \in \mathbb{K}$.

Definition 4.4. An intuitionistic fuzzy topology $\tau$ on $V$ is called an IF vector topology if the intuitionistic fuzzy proper functions $F^{\oplus}:(V \times V, \tau \times \tau) \rightarrow(V, \tau)$ and $F^{\odot}:(\mathbb{K} \times V, \tau \times \nu) \rightarrow$ $(V, \tau)$ are intuitionistic fuzzy continuous. The pair $(V, \tau)$ is said to be an IF topological vector space if $\tau$ is an IF vector topology on $V$.

Remark 4.5. Here we use the term IF topological vector space as there is a notion of intuitionistic fuzzy topological vector space in [18] where the intuitionistic fuzzy topology is in the sense of Coker and the underlying vector space is crisp vector space.

Proposition 4.6. An intuitionistic fuzzy topology $\tau$ on $V$ is an IF vector topology if and only if the intuitionistic fuzzy proper function $F_{V}^{L_{(k, m)}}:(V \times V, \tau \times \tau) \rightarrow(V, \tau)$ is intuitionistic fuzzy continuous.

Proof. Let $\tau$ be an IF vector topology on $V$ and $k, m \in \mathbb{K}$. Since $k \in \mathbb{K}$ is normal element of $\mathbb{K}$ with respect to $V$, by Lemma 3.23 , the intuitionistic fuzzy proper function $F_{k}:(V, \tau) \rightarrow$ $(\mathbb{K} \times V, \nu \times \tau)$ defined by $F_{k}\left(x,\left(k_{1}, x_{1}\right)\right)= \begin{cases}V(x), & \text { if }\left(k_{1}, x_{1}\right)=(k, x) \\ (0,1), & \text { if }\left(k_{1}, x_{1}\right) \neq(k, x)\end{cases}$ is intuitionistic fuzzy continuous. 
Also, by definition of IF vector topology, $F^{\odot}:(\mathbb{K} \times V, \nu \times \tau) \rightarrow(V, \tau)$ is intuitionistic fuzzy continuous.

Hence by Proposition 3.14, $F^{\odot} \circ F_{k}:(V, \tau) \rightarrow(V, \tau)$ defined by

$F^{\odot} \circ F_{k}(x, y)= \begin{cases}V(x) & \text { if } y=k x, k \neq 0 \\ \sup _{s \in X} V(s) & \text { if } y=k x, k=0 \\ (0,1) & \text { otherwise }\end{cases}$

is intuitionistic fuzzy continuous.

Similarly, $F^{\odot} \circ F_{m}:(V, \tau) \rightarrow(V, \tau)$ defined by

$F^{\odot} \circ F_{k}(z, t)= \begin{cases}V(z) & \text { if } t=m x, m \neq 0 \\ \sup _{s \in X} V(s) & \text { if } t=m z, m=0 \\ (0,1) & \text { otherwise }\end{cases}$

is intuitionistic fuzzy continuous.

Thus by Theorem 3.24, $\left(F^{\odot} \circ F_{k}\right) \times\left(F^{\odot} \circ F_{m}\right):(V \times V, \tau \times \tau) \rightarrow(V \times V, \tau \times \tau)$ defined by $\left(F^{\odot} \circ F_{k}\right) \times\left(F^{\odot} \circ F_{m}\right)((x, z),(y, t))= \begin{cases}(V \times V)(x, z) & \text { if }(x, z)=(y, t) \\ (0,1) & \text { if }(x, z) \neq(y, t)\end{cases}$

is intuitionistic fuzzy continuous. Therefore by Proposition 3.14, $F^{\oplus} \circ\left[\left(F^{\odot} \circ F_{k}\right) \times\left(F^{\odot} \circ F_{m}\right)\right]=$ $F_{V}^{L_{(k, m)}}$ is intuitionistic fuzzy continuous.

Conversely, let $F_{V}^{L_{(k, m)}}$ is intuitionistic fuzzy continuous for all $k, m \in \mathbb{K}$.

We know that the projection mapping $p_{V}:(\mathbb{K} \times V, \nu \times \tau) \rightarrow(V, \tau)$ defined by $p_{V}((k, x), z)= \begin{cases}(\mathbb{K} \times V)(k, x) & \text { if } z=x \\ (0,1) & \text { if } z \neq x\end{cases}$

and since $\theta$ is normal of $V$ with respect to $V$, by Lemma 3.22,

$F_{\theta}:(V, \tau) \rightarrow(V \times V, \tau \times \tau)$ defined by

$F_{\theta}\left(x,\left(x_{1}, y_{1}\right)\right)= \begin{cases}V(x) & \text { if }\left(x_{1}, y_{1}\right)=(x, \theta) \\ (0,1) & \text { if }\left(x_{1}, y_{1}\right) \neq(x, \theta)\end{cases}$

are intuitionistic fuzzy continuous proper functions.

Hence by Proposition 3.14, $F_{\theta} \circ p_{V}:(\mathbb{K} \times V, \nu \times \tau) \rightarrow(V \times V, \tau \times \tau)$ defined by

$F_{\theta} \circ p_{V}\left((k, x),\left(x_{1}, y_{1}\right)\right)= \begin{cases}(\mathbb{K} \times V)(k, x) & \text { if }\left(x_{1}, y_{1}\right)=(x, \theta) \\ (0,1) & \text { if }\left(x_{1}, y_{1}\right) \neq(x, \theta)\end{cases}$

is intuitionistic fuzzy continuous.

Therefore $F^{\odot}=\left(F^{L_{(k, 0)}} \circ F_{\theta} \circ p_{V}\right):(\mathbb{K} \times V, \nu \times \tau) \rightarrow(V, \tau)$, where

$\left(F^{L_{(k, 0)}} \circ F_{\theta} \circ p_{V}\right)((k, x), z)= \begin{cases}(\mathbb{K} \times V)(k, x) & \text { if } z=k x, k \neq 0 \\ \sup _{s \in X} V(s) & \text { if } z=k x, k=0 \\ (0,1) & \text { if } z \neq k x\end{cases}$

is intuitionistic fuzzy continuous.

Since $F^{L_{(k, m)}}$ is intuitionistic fuzzy continuous for all $k, m \in \mathbb{K}$, taking $k=1, m=1$ we have $F^{\oplus}:(V \times V, \tau \times \tau) \rightarrow(V, \tau)$ is intuitionistic fuzzy continuous. Hence proved. 
Proposition 4.7. If $(V, \tau)$ is an IF topological vector space, then $F^{k}$ is an intuitionistic fuzzy homeomorphism of $(V, \tau)$ onto itself, for all $k(\neq 0) \in \mathbb{K}$.

Proof. Since $(V, \tau)$ is an $\mathbb{I F}$ topological vector space, $F^{\odot}:(\mathbb{K} \times V, \nu \times \tau) \rightarrow(V, \tau)$ is intuitionistic fuzzy continuous.

Also, by Lemma 3.23, the intuitionistic fuzzy proper function $F_{k}:(V, \tau) \rightarrow(\mathbb{K} \times V, \nu \times \tau)$ defined by $F_{k}\left(x,\left(k_{1}, x_{1}\right)\right)= \begin{cases}V(x), & \text { if }\left(k_{1}, x_{1}\right)=(k, x) \\ (0,1), & \text { if }\left(k_{1}, x_{1}\right) \neq(k, x)\end{cases}$

is intuitionistic fuzzy continuous.

Hence for $k \neq 0, F^{\odot} \circ F_{k}=F^{k}:(V, \tau) \rightarrow(V, \tau)$ defined by

$F^{k}(x, y)= \begin{cases}V(x) & \text { if } y=k x, \\ (0,1) & \text { if } y \neq k x\end{cases}$

is intuitionistic fuzzy continuous.

Similarly, for $k \neq 0$, the intuitionistic fuzzy proper function $\left(F^{k}\right)^{-1}:(V, \tau) \rightarrow(V, \tau)$ defined by $\left(F^{k}\right)^{-1}(x, y)= \begin{cases}V(x) & \text { if } y=\frac{1}{k} x, \\ (0,1) & \text { if } y \neq \frac{1}{k} x\end{cases}$

is intuitionistic fuzzy continuous.

Also $F^{k} \circ\left(F^{k}\right)^{-1}=I_{V}=\left(F^{k}\right)^{-1} \circ F^{k}$. Hence $F^{k}$ is an intuitionistic fuzzy homeomorphism of $(V, \tau)$ onto itself for $k(\neq 0) \in \mathbb{K}$.

Proposition 4.8. If $(V, \tau)$ is an IF topological vector space and if ${ }^{\prime} a^{\prime}$ is a normal element of $V$ with respect to $V$, then $F^{a}$ is an intuitionistic fuzzy homeomorphism of $(V, \tau)$ onto itself.

Proof. If ' $a$ ' is a normal element of $V$ with respect to $V$, then $F^{a}=F^{\oplus} \circ F_{a}$ is intuitionistic fuzzy continuous by continuity of $F^{\oplus}$ and $F_{a}$. Also, inverse of $F^{a}$ is $F^{-a}$ and hence $F^{-a}$ is also intuitionistic fuzzy continuous. Therefore $F^{a}$ is intuitionistic fuzzy homeomorphism from $(V, \tau)$ into itself for any normal ' $a$ ' of $V$ with respect to $V$.

Let $V$ and $W$ be two intuitionistic fuzzy vector spaces in two vector spaces $X$ and $Y$ respectively and $\theta, \theta^{\prime}$ be the null vectors of $X$ and $Y$ respectively.

Definition 4.9. An intuitionistic fuzzy proper function $F: V \rightarrow W$ is said to be an intuitionistic fuzzy linear transformation if

(i) if $F\left(\theta, \theta^{\prime}\right)=\sup _{(x, y) \in(X \times Y)} F(x, y)$,

(ii) $F(k x, k y)= \begin{cases}F(x, y) & \text { if } k \neq 0 \\ \sup _{(x, y) \in(X \times Y)} F(x, y) & \text { if } k=0,\end{cases}$

(iii) if $F(k x, k y)=V(k x)$ and $F(m z, m w)=V(m z)$ imply $F(k x+m z, k y+m w)=V(k x+$ $m z)$,

for all $x, z \in X, y, w \in Y$ and $k, m \in \mathbb{K}$. 
Proposition 4.10. Let $F: V \rightarrow W$ be an intuitionistic fuzzy linear transformation. Then

(i) $F^{-1}(W)$ is an intuitionistic fuzzy vector space over $X$.

(ii) $F(V)$ is an intuitionistic fuzzy vector space over $Y$.

Proof. (i) For any $x \in X$,

$\left[F^{-1}(W)+F^{-1}(W)\right](x)=\underset{x=y+z}{\sqcup}\left\{\left[F^{-1}(W)(y)\right] \sqcap\left[F^{-1}(W)(z)\right]\right\}$

$=\underset{x=y+z}{\sqcup}\left\{V(y) \sqcap W\left(t_{y}\right) \sqcap V(z) \sqcap W\left(t_{z}\right)\right\}$, for $t_{y}, t_{z} \in Y$ such that $F\left(y, t_{y}\right)=V(y)$ and

$F\left(z, t_{z}\right)=V(z)$

$=\underset{x=y+z}{\sqcup}\left\{(V(y) \sqcap V(z)) \sqcap\left(W\left(t_{y}\right) \sqcap W\left(t_{z}\right)\right)\right\}$

$\leq \underset{x=y+z}{\sqcup}\left\{V(y+z) \sqcap W\left(t_{y}+t_{z}\right)\right\}$ [as $V, W$ are intuitionistic fuzzy vector spaces]

$=\underset{x=y+z}{\sqcup}\left\{F\left(y+z, t_{y}+t_{z}\right) \sqcap W\left(t_{y}+t_{z}\right)\right\}$ [Since $F$ is a linear mapping]

$=\underset{x=y+z}{\sqcup}\left\{F\left(x, t_{y}+t_{z}\right) \sqcap W\left(t_{y}+t_{z}\right)\right\}$

$= \begin{cases}V(x) \sqcap W\left(t_{x}\right), & \text { for } t_{x} \in Y \text { unique such that } F\left(x, t_{x}\right)=V(x) \\ (0,1), & \text { otherwise }\end{cases}$

$=F^{-1}(W)(x)$.

Hence $F^{-1}(W)+F^{-1}(W) \subseteq F^{-1}(W)$.

For $k \neq 0,\left[k F^{-1}(W)\right](x)=V\left(\frac{x}{k}\right) \sqcap W\left(y_{\frac{x}{k}}\right)$, for $y_{\frac{x}{k}} \in Y$ unique such that $F\left(\frac{x}{k}, y_{\frac{x}{k}}\right)=V\left(\frac{x}{k}\right)$

$=V\left(x, k y_{\frac{x}{k}}\right)$, since $F$ is linear $F\left(x, k y_{\frac{x}{k}}\right)=F\left(\frac{x}{k}, y_{\frac{x}{k}}\right)$

$=\left[F^{-1}(W)\right](x)$, for all $x \in X$.

For $k=0, x \neq \theta,\left[k F^{-1}(W)\right](x)=(0,1) \leq\left[F^{-1}(W)\right](x)$.

Again for $k=0, x=\theta,\left[k F^{-1}(W)\right](\theta)=\sqcup_{s \in X}\left[F^{-1}(W)(s)\right]$

$=\underset{s \in X}{\sqcup}\left\{\sqcup_{t \in Y}\{F(s, t) \sqcap W(t)\}\right\}$

$\leq[\underset{(s, t) \in(X \times Y)}{\sqcup} F(s, t)] \sqcap\left[\sqcup \sqcup_{t \in Y} W(t)\right]$

$=F\left(\theta, \theta^{\prime}\right) \sqcap\left\{\sqcup_{t \in Y} W(t)\right\}$ [Since $F$ is linear]

$=F\left(\theta, \theta^{\prime}\right) \sqcap W\left(\theta^{\prime}\right)$.

Again $\left[F^{-1}(W)\right](\theta)=\sqcup_{t \in Y}\{F(\theta, t) \sqcap W(t)\}=F\left(\theta, \theta^{\prime}\right) \sqcap W\left(\theta^{\prime}\right)$, [Since $F$ is linear].

Therefore $k F^{-1}(W) \subseteq F^{-1}(W)$, for all $k \in \mathbb{K}$.

Hence $(i)$ is proved.

(ii) For any $z \in Y$,

$[F(V)+F(V)](z)=\underset{z=x+y}{\sqcup}\{[F(V)(x)] \sqcap[F(V)(y)]\}$

$=\underset{z=x+y}{\sqcup}\left\{\left[\sqcup_{t \in X}\{F(t, x) \sqcap V(t)\}\right] \sqcap\left[\sqcup_{s \in X}\{F(s, y) \sqcap V(s)\}\right]\right\}$,

$=\underset{z=x+y}{\sqcup}\left\{\sqcup\left\{V\left(t^{\prime}\right) \sqcap V\left(s^{\prime}\right): t^{\prime}, s^{\prime} \in X\right.\right.$ such that $\left.\left.F\left(t^{\prime}, x\right)=V\left(t^{\prime}\right), F\left(s^{\prime}, y\right)=V\left(s^{\prime}\right)\right\}\right\}$

$\leq \underset{z=x+y}{\sqcup}\left\{\sqcup\left\{V\left(t^{\prime}+s^{\prime}\right): t^{\prime}, s^{\prime} \in X\right.\right.$ such that $\left.\left.F\left(t^{\prime}, x\right)=V\left(t^{\prime}\right), F\left(s^{\prime}, y\right)=V\left(s^{\prime}\right)\right\}\right\}$ as $V \in$ $\operatorname{IFV} S(X)]$

$=\underset{z=x+y}{\sqcup}\left\{\sqcup\left\{V\left(t^{\prime}+s^{\prime}\right): t^{\prime}, s^{\prime} \in X\right.\right.$ such that $\left.\left.F\left(t^{\prime}+s^{\prime}, x+y\right)=F\left(t^{\prime}+s^{\prime}, z\right)=V\left(t^{\prime}+s^{\prime}\right)\right\}\right\}$,

[Since $F$ is a linear].

Now, $F(V)(z)$ 
$=\underset{t \in X}{\sqcup}\{F(t, z) \sqcap V(t)\}$

$=\sqcup\left\{V\left(t_{1}\right): t_{1} \in X, F\left(t_{1}, z\right)=V\left(t_{1}\right)\right\}$.

Hence $F(V)+F(V) \subseteq F(V)$.

For any scalar $k \neq 0, z \in Y$,

$[k F(V)](z)=F(V)\left(\frac{z}{k}\right)$

$=\underset{t \in X}{\sqcup}\left\{F\left(t, \frac{z}{k}\right) \sqcap V(t)\right\}$

$=\sqcup\left\{V(t): t \in X\right.$ such that $\left.F\left(t, \frac{z}{k}\right)=V(t)\right\}$

$=\sqcup\{V(k t): t \in X$ such that $F(k t, z)=V(k t)\}$, [since $V \in I F V S(X)$ and $F$ is linear]

$\leq \underset{s \in X}{\sqcup}\{F(s, z) \sqcap V(s)\}$

$=F(V)(z)$

If $k=0, z \neq \theta^{\prime}$, then

$[k F(V)](z)=(0,1) \leq F(V)(z)$.

Again if $k=0, z=\theta^{\prime}$, then

$[k F(V)]\left(\theta^{\prime}\right)=\underset{y \in Y}{\sqcup}\{F(V)(y)\}$

$=\underset{y \in Y}{\sqcup}\{\underset{x \in X}{\sqcup}[F(x, y) \sqcap V(x)]\}$

$\leq[\underset{(x, y) \in X \times Y}{\sqcup} F(x, y)] \sqcap\left[\sqcup_{x \in X} V(x)\right]$

$=F\left(\theta, \theta^{\prime}\right) \sqcap V(\theta)$, [since $F$ is linear]

Now, $[F(V)]\left(\theta^{\prime}\right)=\sqcup_{x \in X}\left[F\left(x, \theta^{\prime}\right) \sqcap V(x)\right]=F\left(\theta, \theta^{\prime}\right) \sqcap V(\theta)$, [since $F$ is linear].

Therefore $k F(V) \subseteq F(V)$, for all $k \in \mathbb{K}$.

Hence $(i i)$ is proved.

Proposition 4.11. Let $F: V \rightarrow W$ be an intuitionistic fuzzy linear transformation. If $\sigma$ be an IF vector topology on $W$, then $\tau=\left\{F^{-1}\left(W_{1}\right): W_{1} \in \sigma\right\}$ is an IF vector topology on $V$.

Proof. Obviously $\tau$ is an intuitionistic fuzzy topology on $V$. Let $V_{1} \in \tau$. Then there exists $W_{1} \in \sigma$ such that $V_{1}=F^{-1}\left(W_{1}\right)$.

Since $F:(V, \tau) \rightarrow(W, \sigma)$ is intuitionistic fuzzy continuous, $F \times F:(V \times V, \tau \times \tau) \rightarrow$ $(W \times W, \sigma \times \sigma)$ is also so.

Again since $(W, \sigma)$ is an IF topological vector space $F_{W}^{L_{(k, m)}}:(W \times W, \sigma \times \sigma) \rightarrow(W, \sigma)$ is intuitionistic fuzzy continuous, hence $\left(F_{W}^{L_{(k, m)}}\right)^{-1}\left(W_{1}\right) \in \sigma \times \sigma$ and so, $(F \times F)^{-1}\left(\left(F_{W}^{L_{(k, m)}}\right)^{-1}\left(W_{1}\right)\right) \in$ $\tau \times \tau$.

Now, $(F \times F)^{-1}\left(\left(F_{W}^{L_{(k, m)}}\right)^{-1}\left(W_{1}\right)\right)\left(x_{1}, x_{2}\right)$

$=(V \times V)\left(x_{1}, x_{2}\right) \sqcap\left[\left(F_{W}^{L_{(k, m)}}\right)^{-1}\left(W_{1}\right)\right]\left(y_{1}, y_{2}\right)$, where $F\left(x_{i}, y_{i}\right)=V\left(x_{i}\right)$, for $i=1,2$

$=(V \times V)\left(x_{1}, x_{2}\right) \sqcap(W \times W)\left(y_{1}, y_{2}\right) \sqcap W_{1}\left(k y_{1}+m y_{2}\right)$

$=(V \times V)\left(x_{1}, x_{2}\right) \sqcap W_{1}\left(k y_{1}+m y_{2}\right)$, [since $V\left(x_{i}\right) \leq W\left(y_{i}\right)$, for $\left.i=1,2\right] \ldots . .(I)$

Again, $\left[\left(F_{V}^{L_{(k, m)}}\right)^{-1}\left(V_{1}\right)\right]\left(x_{1}, x_{2}\right)$

$=(V \times V)\left(x_{1}, x_{2}\right) \sqcap\left(V_{1}\right)\left(k x_{1}+m x_{2}\right)$

$=(V \times V)\left(x_{1}, x_{2}\right) \sqcap\left[F^{-1}\left(W_{1}\right)\right]\left(k x_{1}+m x_{2}\right)$

$=(V \times V)\left(x_{1}, x_{2}\right) \sqcap\left[V\left(k x_{1}+m x_{2}\right) \sqcap W_{1}\left(k y_{1}+m y_{2}\right)\right]$, [since $F$ is linear]

$=(V \times V)\left(x_{1}, x_{2}\right) \sqcap W_{1}\left(k y_{1}+m y_{2}\right)$, [since $\left.V \in I F V S(X)\right] \ldots . .(I I)$ 
From $(I)$ and $(I I)$ we have, $\left(F_{V}^{L_{(k, m)}}\right)^{-1}\left(V_{1}\right)=(F \times F)^{-1}\left(\left(F_{W}^{L_{(k, m)}}\right)^{-1}\left(W_{1}\right)\right) \in \tau \times \tau$.

Therefore $(V, \tau)$ is an IF topological vector space.

Proposition 4.12. Let $F: V \rightarrow W$ be an injective intuitionistic fuzzy linear transformation. If $\tau$ is a IF vector topology on $V$, then $\sigma=\left\{W^{\prime} \subseteq W: F^{-1}\left(W^{\prime}\right) \in \tau\right\}$ is an IF vector topology on $F(V)$. If further $F$ is surjective, then $\sigma$ is an IF vector topology on $W$.

Proof. Since $F$ is injective for all $V_{1} \subseteq V, F^{-1}\left(F\left(V_{1}\right)\right)=V_{1}$.

It can be easily verified that $\sigma$ is an intuitionistic fuzzy topology on the vector space $F(V)=W_{1}$ (say). Since $F$ is injective, $F:(V, \tau) \rightarrow\left(W_{1}, \sigma\right)$ is intuitionistic fuzzy open.

Let $W^{\prime} \in \sigma$. Then $F^{-1}\left(W^{\prime}\right) \in \tau$.

Since $\tau$ is an IF vector topology on $V,\left(F_{V}^{L_{(k, m)}}\right)^{-1}\left(F^{-1}\left(W^{\prime}\right)\right) \in \tau \times \tau$.

Since $F \times F:(V \times V, \tau \times \tau) \rightarrow\left(W_{1} \times W_{1}, \sigma \times \sigma\right)$ is intuitionistic fuzzy open, $(F \times$ $F)\left(F_{V}^{L_{(k, m)}}\right)^{-1}\left(F^{-1}\left(W^{\prime}\right)\right) \in \sigma \times \sigma$.

Now $\left(F_{W_{1}}^{L_{(k, m)}}\right)^{-1}\left(W^{\prime}\right)\left(y_{1}, y_{2}\right)$

$=\underset{y_{3} \in Y}{\sqcup}\left\{\left[F_{W_{1}}^{L_{(k, m)}}\left(\left(y_{1}, y_{2}\right), y_{3}\right)\right] \sqcap W^{\prime}\left(y_{3}\right)\right\}$

$=\left(W_{1} \times W_{1}\right)\left(y_{1}, y_{2}\right) \sqcap W^{\prime}\left(k y_{1}+m y_{2}\right)$

$=[F(V) \times F(V)]\left(y_{1}, y_{2}\right) \sqcap F\left(V_{1}\right)\left(k y_{1}+m y_{2}\right)$ [Since $F$ is injective, there is $V_{1} \subseteq V$ such that $\left.F\left(V_{1}\right)=W^{\prime}\right]$.

Again, $F\left(V_{1}\right)\left(k y_{1}+m y_{2}\right)=\underset{t \in X}{\sqcup}\left\{F\left(t, k y_{1}+m y_{2}\right) \sqcap V_{1}(t)\right\}$

$=V\left(t_{1}\right) \sqcap V_{1}\left(t_{1}\right)$, where $t_{1} \in X$ with $F\left(t_{1}, k y_{1}+m y_{2}\right)=V\left(t_{1}\right)$;

$=V_{1}\left(t_{1}\right)$, where $t_{1} \in X$ with $F\left(t_{1}, k y_{1}+m y_{2}\right)=V\left(t_{1}\right)$;

$=V_{1}\left(k x_{1}+m x_{2}\right)$, for $\left(x_{1}, x_{2}\right) \in X \times X$ such that $F\left(x_{i}, y_{i}\right)=V\left(x_{i}\right)$, for $i=1,2$, as $F$ is linear.

Therefore, $\left(F_{W_{1}}^{L_{(k, m)}}\right)^{-1}\left(W^{\prime}\right)\left(y_{1}, y_{2}\right)$

$=(V \times V)\left(x_{1}, x_{2}\right) \sqcap V_{1}\left(k x_{1}+m x_{2}\right)$, where $F\left(x_{i}, y_{i}\right)=V\left(x_{i}\right)$, for $i=1,2 \ldots \ldots \ldots(I I I)$

Again $\left(F_{V}^{L_{(k, m)}}\right)^{-1}\left(F^{-1}\left(W^{\prime}\right)\right)\left(x_{1}, x_{2}\right)$

$=\underset{t \in X}{\sqcup}\left\{F_{V}^{L_{(k, m)}}\left(\left(x_{1}, x_{2}\right), t\right) \sqcap F^{-1}\left(W^{\prime}\right)(t)\right\}$

$=(V \times V)\left(x_{1}, x_{2}\right) \sqcap F^{-1}\left(W^{\prime}\right)\left(k x_{1}+m x_{2}\right)$

$=(V \times V)\left(x_{1}, x_{2}\right) \sqcap V_{1}\left(k x_{1}+m x_{2}\right)$, since $F$ is injective, $F^{-1}\left(W^{\prime}\right)=F^{-1}\left(F\left(V_{1}\right)\right)=V_{1}$.

Hence $(F \times F)\left(F_{V}^{L_{(k, m)}}\right)^{-1}\left(F^{-1}\left(W^{\prime}\right)\right)\left(y_{1}, y_{2}\right)$

$=\underset{(t, s) \in X \times X}{\sqcup}\left\{(F \times F)\left((t, s),\left(y_{1}, y_{2}\right)\right) \sqcap\left(\left(F_{V}^{L_{(k, m)}}\right)^{-1}\left(F^{-1}\left(W^{\prime}\right)\right)(t, s)\right\}\right.$

$=(V \times V)\left(x_{1}, x_{2}\right) \sqcap V_{1}\left(k x_{1}+m x_{2}\right)$, where $F\left(x_{i}, y_{i}\right)=V\left(x_{i}\right)$, for $i=1,2 \ldots \ldots \ldots .(I V)$

Therefore from $(I I I)$ and $(I V)$, we have $\left(F_{W}^{L_{(k, m)}}\right)^{-1}\left(W^{\prime}\right)=(F \times F)\left(F_{V}^{L_{(k, m)}}\right)^{-1}\left(F^{-1}\left(W^{\prime}\right)\right) \in$ $\sigma \times \sigma$ and hence $\sigma$ is an IF vector topology on $F(V)$.

If further $F$ is surjective, then $F(V)=W$. Hence proved.

\section{Acknowledgements}

The research of the 1st author is supported by UGC (University Grants Commission), India under Junior Research Fellowship in Science, Humanities and Social Sciences. 


\section{References}

[1] Atanassov, K. T. (1986) Intuitionistic fuzzy sets, Fuzzy Sets and Systems, 20, 87-96.

[2] Atanassov, K. T. (1994) New operations defined over intuitionistic fuzzy sets, Fuzzy Sets and Systems, 61(2), 137-142.

[3] Atanassov, K. T. (1999) Intuitionistic fuzzy sets - Theory and Applications, Studies in Fuzziness and Soft Computing, Physica-Verlag Heidelberg, 35.

[4] Atanassov, K. T. (2012) On Intuitionistic Fuzzy Sets Theory, Studies in Fuzziness and Soft Computing Series, Vol 283, Springer, Berlin.

[5] Atanassova, L. (2007) On intuitionistic fuzzy versions of L. Zadeh's extension principle, Notes on Intuitionistic Fuzzy Sets,13(3), 33-36.

[6] Biswas, R. (1989) Intuitionistic fuzzy subgroups, Math. Forum, 10, 37-46.

[7] Biswas, R. (1997) On fuzzy sets and intuitionistic fuzzy sets, Notes on Intuitionistic Fuzzy Sets, 3, 3-11.

[8] Chakraborty, M. K., \& Ahsanullah, T. M. G. (1992) Fuzzy topology on fuzzy sets and tolerance topology, Fuzzy Sets and Systems, 45, 103-108.

[9] Chen, Wenjuan, \& Zhang, Shunhua, (2009) Intuitionistic fuzzy Lie sub-superalgebras and intuitionistic fuzzy ideals, Computers and Mathematics with Applications, 58, 1645-1661.

[10] Chiney, M., \& Samanta, S. K. (2017) Intuitionistic fuzzy basis of an intuitionistic fuzzy vector space, Notes on Intuitionistic Fuzzy Sets, 23(4), 62-74.

[11] Chiney, M., \& Samanta, S. K. (2018) Intuitionistic fuzzy dimension of an intuitionistic fuzzy vector space, Notes on Intuitionistic Fuzzy Sets, 24(1), 21-29.

[12] Coker, D., (1997) An introduction to intuitionistic fuzzy topological spaces, Fuzzy Sets and Systems, 88, 81-89.

[13] De, S. K., Biswas, R., \& Roy, A. R. (2001) An application of intuitionistic fuzzy sets in medical diagnostic, Fuzzy sets and systems, 117 (2), 209-213.

[14] Ejegwa, P. A., Akubo, A. J., \& Joshua, O. M. (2014) Intuitionistic fuzzy set and its application in career determination via normalized euclidean distance method, European Scientific Journal, 10(15), 529-536.

[15] Hur, K., Kang, H. W., \& Song, H. K. (2003) Intuitionistic fuzzy subgroups and subrings, Honam Math. J., 25, 19-41.

[16] Katsaras, A. K. (1981) Fuzzy topological vector spaces I, Fuzzy sets and systems, 6, 85-95. 
[17] Lowen, R. (1976) Fuzzy topological spaces and fuzzy compactness, J. Math. Anal. Appl., $56,621-633$.

[18] Mohammed, M. J., \& Ataa, G. A. (2014) On Intuitionistic fuzzy topological vector space, Journal of College of Education for Pure Sciences, 4, 32-51.

[19] Mondal, K. K., \& Samanta, S. K. (2013) A study on Intuitionistic fuzzy topological spaces, Notes on Intuitionistic Fuzzy Sets, 9(1), 1-32.

[20] Park, J. H. (2004) Intuitionistic fuzzy metric spaces, Chaos Solitons Fractals, 22 , 10391046.

[21] Pradhan, R., \& Pal, M. (2012) Intuitionistic fuzzy linear transformations, Annals of Pure and Applied Mathematics, 5(1), 57-68.

[22] Roopkumar R., \& Kalaivani C. (2010) Continuity of intuitionistic fuzzy proper functions on intuitionistic smooth fuzzy topological spaces, Notes on Intuitionistic Fuzzy Sets, 16(3), $1-21$.

[23] Szmidt, E., \& Kacprzyk, J. (1996) Intuitionistic fuzzy sets in group decision making, Notes on Intuitionistic Fuzzy Sets, 2 (1), 11-14.

[24] Zadeh, L.A. (1965) Fuzzy sets, Information and Control 8 338-353. 